\title{
Tuneable Local Structure in Thermoelectric Crystals
}

\author{
N. Roth \\ Department of Chemistry, University of Oxford, Inorganic Chemistry Laboratory, South Parks Road, Oxford, OX1 3QR, UK \\ nikolajroth@gmail.com
}

Crystalline phases are usually characterised by their periodic structures and space group symmetry. However, some crystalline materials have periodic structures only on average and deviate on a local scale. Several different locally ordered structures can exist with identical average periodic structure and space group symmetry, making them difficult to distinguish using regular crystallographic techniques.

Using high-quality single-crystal $\mathrm{x}$-ray diffuse scattering the local order in thermoelectric half-Heusler $\mathrm{Nb}_{1-\mathrm{x}} \mathrm{CoSb}$ is investigated, for which different local orderings are observed. Half-Heusler materials have been intensely studied for their thermoelectric properties, but a general issue is their high thermal conductivity due to their simple structure. The defective half-Heuslers such as $\mathrm{Nb}_{1-\mathrm{x}} \mathrm{CoSb}$ have high vacancy concentrations $(x=1 / 6)$, giving them much lower thermal conductivities than other half-Heusler compounds. From measurements on different samples of $\mathrm{Nb}_{1-\mathrm{x}} \mathrm{CoSb}$, it is shown that crystals with identical stoichiometry and average crystal structure, but with different locally ordered structures, can be made by changing the synthesis method. The local structures in these samples are analysed using the three-dimensional difference pair distribution function (3D- $\Delta \mathrm{PDF})$.

A new method is shown which allows isolation of the substitutional correlations in the $3 \mathrm{D}-\Delta \mathrm{PDF}$, showing that the vacancy distributions follow a vacancy repulsion model. Furthermore, the local structural relaxations around vacancies are quantised from analysis of Bragg peaks and 3D- $\triangle \mathrm{PDF}$. From the found short-range correlations, a physical model of the system is simulated using Monte-Carlo methods, and it is shown that the different samples correspond to the ground state and simulated quenched states of the model.

Advanced x-ray scattering techniques can unravel hidden local structures and for $\mathrm{Nb}_{1-\mathrm{x}} \mathrm{CoSb}$ these local structures can be controlled by the synthesis conditions. If the local structure of crystalline materials can be more generally related to the properties, then a new frontier in materials research will be available.

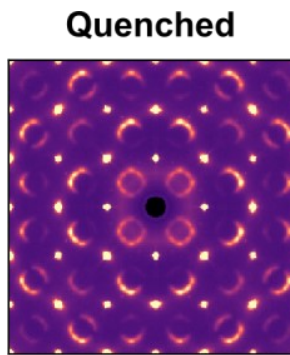

Slowly cooled
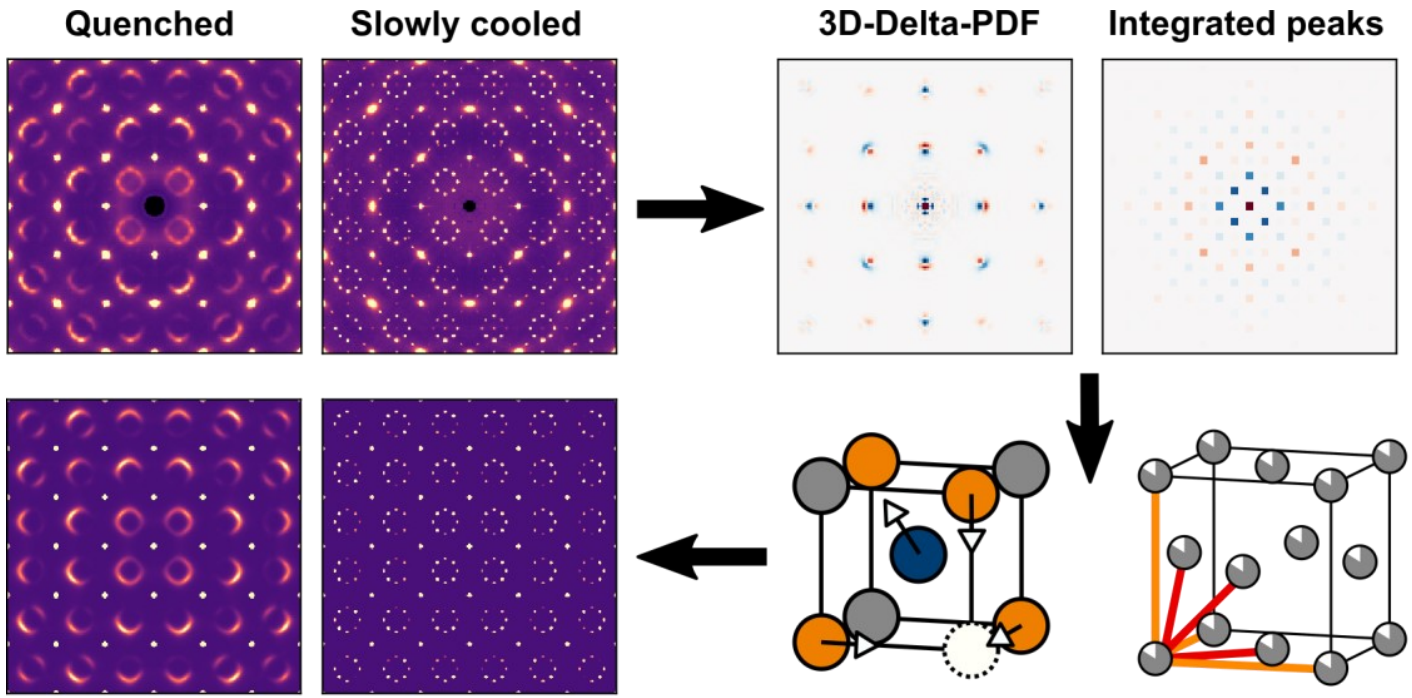

Model simulation

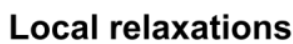

Vacancy repulsion

Figure 1. Measured scattering, measured 3D- $\triangle \mathrm{PDF}$, structural model and simulated scattering for defective half-Heuslers.

[1] Roth, N., et al (2020) IUCrJ. 7, 673-680

[2] Roth, N., et al (2021) arXiv:2103.08543

Keywords: Single-Crystal Diffuse scattering; 3D-delta-PDF; short-range order; Hidden phases; Thermoelectrics 